\title{
BMJ Open What are the physical and psychological health effects of suicide bereavement on family members? Protocol for an observational and interview mixed-methods study in Ireland
}

\author{
Ailbhe Spillane, ${ }^{1,2}$ Celine Larkin, ${ }^{2}$ Paul Corcoran, ${ }^{1,3}$ Karen Matvienko-Sikar, ${ }^{1}$ \\ Ella Arensman ${ }^{1,2}$
}

To cite: Spillane A, Larkin C, Corcoran $\mathrm{P}$, et al. What are the physical and psychological health effects of suicide bereavement on family members? Protocol for an observational and interview mixed-methods study in Ireland. BMJ Open 2017:7:e014707.

doi:10.1136/bmjopen-2016014707

- Prepublication history for this paper is available online. To view these files please visit the journal online (http://dx.doi.org/10.1136/ bmjopen-2016-014707)

Received 12 October 2016 Revised 14 February 2017 Accepted 17 February 2017

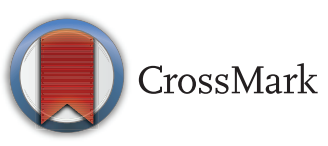

${ }^{1}$ Department of Epidemiology and Public Health, Cork, Ireland

${ }^{2}$ National Suicide Research Foundation, Cork, Ireland ${ }^{3}$ Department of Obstetrics and Gynaecology, Cork University Maternity Hospital, Cork, Ireland

Correspondence to Ailbhe Spillane; ailbhe.spillane@ucc.ie

\section{ABSTRACT}

Introduction: Research indicates that experiencing the suicide of a relative can have a significant impact on family members' emotional health. However, research incorporating the impact of suicide bereavement on family members' physical health is sparse. This paper details the protocol for a mixed-methods study of suicide-bereaved family members. The study will primarily examine the physical and mental health needs of those bereaved by suicide. A secondary objective of the study is to describe the support service needs of family members bereaved by suicide.

Methods and analysis: A mixed-methods approach, using semistructured interviews and self-report questionnaires, will be used. Interviews will be conducted with a group of 15-20 relatives who experienced suicide bereavement. This protocol will follow the COREQ checklist criteria for the reporting of qualitative research interviews. Thematic analysis will be used to examine experiences and impact of bereavement on psychological and physical health. Self-report quantitative data on well-being will be analysed using descriptive statistics.

Ethics and dissemination: Ethical approval to conduct this study has been granted from the Clinical Research Ethics Committee of the Cork Teaching Hospitals. Pseudonyms will be given to participants to protect anonymity. It will be explained to participants that participation in the study is voluntary and they have to right to withdraw at any time. The findings of this research will be disseminated to regional, national and international audiences through publication in peer-reviewed international journals and presentations at scientific conferences. This research also forms part of a $\mathrm{PhD}$ thesis.

\section{INTRODUCTION}

Approximately 800000 people die by suicide annually, resulting in an estimated 48-500 million people experiencing suicide

\section{Strengths and limitations of this study}

- This study addresses a specific gap in the literature by exploring and examining the physical and psychological health effects of suicide bereavement on family members in Ireland.

- The backgrounds and characteristics of family members included in the qualitative component of the study are diverse, thereby allowing for a range of experiences and rich data.

- The inclusion of probable cases of suicide will help to identify 'hidden' suicide cases, which would otherwise not have been included.

- Participants are drawn from a small geographic area and the findings of this study may not be generalisable to other settings. However, the main aim of qualitative research is to be credible and transferrable.

bereavement every year worldwide. ${ }^{1}{ }^{2}$ Suicide and self-harm can have a significant impact on family members' emotional and social functioning. ${ }^{3-6}$ Suicide bereavement is associated with a number of adverse mental health outcomes, including depression, psychiatric admission and suicide attempts. ${ }^{1} 367$ However, research examining the physical health effects of suicide bereavement is sparse.

It is increasingly recognised that negative psychological factors, including depression, are implicated in the development and advancement of cardiovascular disease (CVD). ${ }^{8}{ }^{9}$ Stressful life events also impact physical health, ${ }^{10}$ and the experience of bereavement in particular is associated with negative health outcomes. ${ }^{11}$ Bereavement is not only associated with an excess risk of mortality but also physical ill-health and negative psychological reactions and symptoms, 
including mental disorders or complications related to the grieving process. ${ }^{11}$ There is also emerging evidence of the effect of suicide bereavement on physical health: for example, a recent case-control study found that suicide-bereaved parents have a higher risk of CVD, hypertension, diabetes and chronic obstructive pulmonary disease (COPD). ${ }^{6}$ Given the increased risk of poor health among those experiencing bereavement and mental health problems, more research is required into the physical health sequelae of suicide bereavement. The majority of extant studies on this topic are quantitative in nature. ${ }^{6}{ }^{12-15}$ Therefore, qualitative research is required to fully understand and effectively respond to the needs experienced by people affected by the suicide of their family member. ${ }^{16}$

\section{The impact of suicide on the individual, family and social life}

People bereaved by suicide are at increased risk of negative physical outcomes, including CVD, COPD, hypertension, diabetes and pancreatic cancer. ${ }^{6} 7$ Nevertheless, these findings are only evident from a small number of empirical studies, with much of the research focusing on mental health outcomes following suicide bereavement. ${ }^{13}$ People bereaved by suicide are also at increased risk of suicide, depression and psychiatric admission as has been demonstrated by the findings of populationbased registers/registry studies. ${ }^{16}{ }^{17}$ In terms of findings related to substance use, two systematic reviews did not find any excess risk of substance misuse among people bereaved by suicide, ${ }^{11}{ }^{18}$ while another case-control study did not find any differences in substance misuse between suicide and accident-bereaved parents. ${ }^{6}$ Even though one study found an increased risk in offspring bereaved by suicide, this analysis was not adjusted for preloss substance misuse. ${ }^{17}$ Recent systematic reviews found that studies comparing stigma scores were methodologically problematic due to unadjusted analyses and that when research was specifically comparing suicide bereavement and other violent causes of death, the only differences noted were on rejection and shame. ${ }^{1}{ }^{19}$ Notably, a large cross-sectional study in the UK found significantly higher levels of stigma, shame, guilt and responsibility in people bereaved by suicide compared with those bereaved by other sudden deaths in adjusted analyses. $^{20}$

\section{Existing qualitative studies}

Although there are a number of qualitative studies examining various aspects of suicide bereavement, including experiences, ${ }^{21}$ stigma $^{22}$ and sense/meaningmaking, ${ }^{23}$ so far no study has examined the physical and psychological experiences and support needs of people bereaved by suicide.

Subsequent to suicide bereavement, parents hiding their grief and emotions is a common theme throughout the literature. ${ }^{21}{ }^{24}$ Fear of judgement and stigmatisation was the most common reason cited for not seeking professional support following a suicide bereavement. ${ }^{25}$ Similarly, parents describe struggling to speak about their child in public, as they felt it is frowned on and is too morbid. ${ }^{24}$ This avoidance of grief-related emotions may prevent relatives from engaging in meaningmaking, whereby they assimilate the death of their loved one into their new reality. ${ }^{26} \mathrm{~A}$ lack of engagement in meaning-making may increase the risk of developing complicated grief. ${ }^{24} 2728$ While bereavement due to suicide and sudden death can be devastating for families, adjustment to the death is still possible. Talking about the deceased is thought to be an important part of the grief process and some parents have found it valuable to recall the deceased as he/she had been. ${ }^{29}$ An interpretative phenomenological study identified that adults bereaved by suicide can undergo post-traumatic growth (PTG).$^{30}$ PTG can be defined as heightened levels of personal development reached in the aftermath of trauma ${ }^{31}$ and is associated with better mental health outcomes in people bereaved by suicide. ${ }^{32}$ People bereaved by suicide undergoing this process report developing greater awareness of their existence in the world and understanding what mortality means to them, facilitating a desire to make the most out of life. ${ }^{30}$ PTG should be normalised, so that the suicide bereaved can explore how they have altered and changed without feeling further stigmatised or judged. ${ }^{30}$ Sometimes, bereaved people may experience relief ${ }^{33}$ following a family member's suicide if there was ongoing mental health and/or physical health problems over many years. It is therefore essential to understand the complex and sometimes contradictory experiences of suicide bereavement and grief in order to better help people express their emotions and potentially promote PTG.

\section{Current study}

It is clear that suicide bereavement has an impact on health and mortality, with those affected at increased risk of suicide and psychiatric admission, ${ }^{16}$ yet most of this research is quantitative in nature. A more nuanced understanding of why these help problems arise is necessary. Qualitative research would best help to explain some of the mechanisms of poor health outcomes. In addition, qualitative methodologies will help develop a better understanding of the perspectives of the individual family members. ${ }^{16}$ The current mixed-methods study, which is a follow-up to the Suicide Support and Information System-A Case-control Study (SSIS-ACE), will address this gap in the literature. The aim of the SSIS-ACE study is to improve knowledge surrounding the specific psychosocial, psychiatric and work-related risk factors associated with suicide in Ireland. The SSIS-ACE study incorporates two interlinked casecontrol studies: one study comparing suicide cases with general practice patient controls, and the other study comparing cases of high-risk suicidal behaviour with general practice patient controls. The SSIS-ACE study 
included suicide decedents as cases and relatives as informants to better understand the decedents risk and protective factors through a psychological autopsy interview approach. The current study includes relatives as cases to understand how being bereaved by suicide or an open verdict death has impacted them physically and emotionally. Therefore, the focus of the SSIS-ACE study was on the deceased, whereas the focus of the present study is on the family member and how they have been affected by the death. The primary aim of the current study is to follow-up relatives bereaved by suicide who had been recruited for the SSIS-ACE study, to examine how family members have been physically and psychologically affected by a relative's suicide. A secondary objective of the study is to describe the support required by family members bereaved by suicide. Specific objectives for this study are as follows:

1. To understand the pathways by which physical and mental health problems might arise in family members bereaved by suicide.

2. To examine the broader familial and social impact of suicide bereavement for family members.

3. To examine participants' experiences of health and support services and to understand what service provisions they consider important for the needs of family members after a suicide bereavement.

4. To assess emotional well-being among family members following suicide bereavement.

\section{METHODS AND ANALYSIS}

\section{Theoretical approach}

Pragmatism was chosen as the most appropriate philosophical underpinning for this study. Pragmatism aids in understanding how quantitative and qualitative methods can be combined successfully to best answer particular research questions. ${ }^{34}$ Pragmatism was chosen as an approach for the current study, as it was anticipated that multiple methods would be required in order to fully address the research objectives. A social constructionist perspective was adopted as the theoretical perspective for analysis in the current study. This theory posits that social processes underlie or are the building blocks for how one understands and interprets the world. It is further acknowledged that this perspective sees reality as something that is dependent on what knowledge one is exposed to through social, historical or political interactions. ${ }^{35}$ One must begin a process of meaning-making and a reconstruction of a new reality following a loss. ${ }^{36}$ This process can be especially difficult for family members bereaved by suicide due to unique factors associated with suicide bereavement. In summary, therefore it is critical to acknowledge and understand participants' social reality and how they have come to understand this reality as this can have a significant bearing on how adaptive or maladaptive their grief reactions become, thereby affecting psychological and physical health outcomes.

\section{Study design and setting}

This exploratory mixed-methods study is a follow-up to a larger case-control study, which is examining the psychological, psychiatric and work-related factors associated with suicide in Ireland (SSIS-ACE, 2014-2017). The SSIS-ACE study began in January 2014 and will be completed by March 2017. The current study began in April 2016 and is envisaged to be completed by April 2017. Participants for the qualitative aspect of this study are drawn from the larger SSIS-ACE study. Interviews are conducted either at the offices of the National Suicide Research Foundation (NSRF) in Cork, in the participant's home or in a neutral location. Only the researcher and the participant are present during the interviews. The location of the interview is determined by the participants according to their preference.

\section{Sampling}

All participants who completed the SSIS-ACE interview and who consented to further follow-up are invited to take part in the follow-up study. There was no relationship established prior to the initiation of the study. Participants were originally approached to participate in the SSIS-ACE study on the basis of being next-of-kin of persons who died by suicide or probable suicide. It is not unusual in suicide research to consider open verdict deaths, as research suggests a substantial number of these are thought to be suicides. ${ }^{37-39}$ Open (undetermined deaths) and narrative verdicts that met the Rosenberg criteria ${ }^{40}$ for a probable suicide are eligible for inclusion in the current study. The Rosenberg criteria for the determination of suicide state that 'a death must have been self-inflicted, which may be determined by a number of means including autopsy, toxicology, psychological evidence and decedent and witness testimony'. Second, there must be evidence that the person intended to kill him/herself and understood the outcome of the action. Evidence of intent can include explicit verbal or non-verbal expressions of intent to take one's life but can also include implicit or indirect expressions of intent to kill oneself, including: ${ }^{40}$

- Inappropriate or unexpected preparations for death by the deceased,

- Expressing farewell or expressing a desire to die or acknowledging impending death,

- Expression of hopelessness,

- Expression of significant emotional or physical pain or distress,

- Efforts to learn about or procure means of death or to rehearse fatal behaviour,

- Precautions to avoid rescue,

- Evidence that decedent recognised high-potential lethality of means of death,

- Previous suicide attempt,

- Previous threat of suicide,

- Stressful events or significant losses (actual or threatened), or

- Serious depression or mental disorder. 
A potential participant is excluded if they are aged younger than 18 years, if contact is deemed to pose a risk to the safety of the researcher (likely intoxication; history of or potential for violence) or the informant, or where capacity to consent may be limited due to cognitive dysfunction or severe mental illness. Recruitment will proceed to the point of data saturation, which is envisaged to involve interviewing and analysing transcripts from 15 to 20 participants. The use of eligibility criteria (box 1) together with the inclusion of consecutive cases of suicide and probable suicide will provide a diverse sample of people bereaved by suicide.

\section{Recruitment}

As of April 2016, participants bereaved by suicide, who previously took part in the SSIS-ACE study, were invited by letter to participate. The letter explains that the researcher will contact the family member again 10 days after receipt of the letter to verify if there is a need for support and to provide further details about the study. It is clearly stated that the family member can inform the researcher (in writing or by telephone) if they do not wish to receive any further contact. The focus of the first telephone contact is to verify again if the family members would like to receive support. AS facilitated access to support for participants based on their specific needs. This is performed in consultation with a trained psychotherapist (PI, EA). The researcher also verifies if currently there are members of the family who are in need of support that has not yet been arranged. AS will contact each participant by phone 2-3 weeks after the conclusion of the interview to discuss any reflections or needs for support they may have following the interview.

\section{Well-being (Depression Anxiety and Stress Scale) scale}

To assess family member's affective state, their wellbeing has been assessed using the 21-item version of the Depression Anxiety and Stress Scale (DASS). ${ }^{41}$ These

Box 1 Eligibility criteria for the selection of family members bereaved by suicide/open verdict

\section{Inclusion criteria}

1. Must be aged 18 years or older

2. Must have consented for future contact from research team during the larger case-control study

3. Must have experienced the suicide/undetermined death of a relative

Exclusion criteria

1. If contact is deemed to pose a risk to the safety of the researcher (likely intoxication; history of or potential for violence) or the informant

2. If capacity to consent is limited due to cognitive dysfunction or severe mental illness, identified via collaborating with coroners and members of the police force when necessary data were collected as part of the SSIS-ACE study, where data collection occurred between June 2014 and September 2016. These data will be presented together with the qualitative interview data in the full publication in order to provide mental health, physical health and well-being outcomes for family members bereaved by suicide. The depression subscale covers dysphoria, hopelessness, devaluation of life, self-deprecation, lack of interest/involvement, anhedonia and inertia. Items related to anxiety are autonomic arousal, skeletal musculature effects, situational anxiety and subjective experience of anxious effect. Finally, items related to stress are difficulty relaxing, nervous arousal, being easily upset, irritability and impatience. ${ }^{41}$ This scale was completed at the end of the interview with the SSIS-ACE study. The DASS scale has been shown to successfully differentiate between the three negative affective states. ${ }^{41}$ In addition, this scale is consistent in clinical (those presenting themselves to anxiety and stress clinics for assessment and treatment) and non-clinical (community volunteers) samples. ${ }^{41}$ The scale has also been demonstrated to have excellent internal consistency in a large clinical sample, with the following Cronbach's $\alpha$ value of $0.96,0.89$ and 0.93 for Depression, Anxiety and Stress, respectively. ${ }^{42}$ Another study of a clinical sample found similar Cronbach's $\alpha$ values of $0.97,0.92$ and 0.95 for Depression, Anxiety and Stress, respectively. ${ }^{43}$

\section{Interviews}

Interviews were started in April 2016. Semistructured interviews, with the use of a topic guide (table 1), were conducted. Interviews were conducted in one sitting. These data will help to provide a comprehensive description of the sample in the full publication. Demographic data collected during the interview include gender, age, relationship to the deceased, ethnic origin, religion, marital status, employment status, education level and profession. Before the initiation of each interview, AS explained that the current research is essential due to the limited research conducted into the physical and psychological health effects of suicide and sudden death bereavement on family members in Ireland. The duration of each interview was $\sim 1.5-2$ hours. The aim of the interviews is to gain an indepth understanding of the personal, familial and social impact of suicide bereavement on family members. The topic guide serves to guide the interviews, while not being prescriptive in nature. This allows scope to iteratively develop the topic guide in order to deal with issues that are most relevant to participants.

Participants were contacted 24 hours prior to the interview to ascertain if it was still convenient to meet with AS. Interviews were audio-recorded with permission of the participant. Field notes and memos were taken in order to provide context or to document initial thoughts or emerging themes identified by the interviewer. Characteristics and reasons of those who decline 
Table 1 Topic guide for family members bereaved by suicide/undetermined deaths

\section{Prompts}

Introduction

- Family member's relationship with deceased

How did you spend time together? What was that like for you?

Impact of bereavement on family member

- How they felt physically in the immediate aftermath of relative's death

- How their physical health was before and since their relative's death

Were there any physical symptoms? How did this affect you physically?

Presence of or new diagnosis of health condition? Worsening of existing health condition? Changes in diet, exercise, smoking, sleeping patterns or substance use?

- How their mental health was before and since their relative's death

- How has their sense of well-being been since their relative's death

- Had deceased ever harmed themselves before?

- How did that make them feel?

Feelings of stress or worry? New diagnosis of health condition?

Worsening of existing health condition?

Experiencing positive thoughts? Experiencing negative thoughts?

Optimism regarding the future?

Were there any physical symptoms? How did this affect you physically?

\section{Familial and social impact of bereavement}

- How the death has affected their life

- How the death has affected the family

Social life/work life? Outlook on life?

- How any physical illnesses experienced by the family member after the death has affected their social/work life

\section{Postvention}

-What supports they received from additional family and friends after the death of the family member

- Any support services they encountered, whether they found it helpful/unhelpful and why

- Any particular service they would like to see in immediate aftermath of relative's death

- Any particular service they would like to see in medium to long term following relative's death

- Do they have a message for:

- Healthcare professionals?

- Mental health services or counsellors?

- Others who have been bereaved?

- People contemplating suicide?

Anything to add before conclusion of interview?

to participate were noted and will be reported in the full publication.

Data management (memos, field notes, verbatim transcription, etc)

Interviews were transcribed verbatim by AS and other internal research staff. All participants were informed that an additional researcher may listen to the recording but are not provided with any additional information or have subsequent involvement in the study. The names of participants were replaced with pseudonyms when each interview was transcribed. Field notes were taken after interviews, which will facilitate the analysis process. A reflective journal was used to convey the main issues raised in the interviews, and served as preliminary analysis of the data.

\section{Data analysis}

Quantitative analysis

Participant's demographic information and descriptive statistics, including mean scores, will be calculated for the well-being data and will be presented in the full publication.

\section{Qualitative analysis}

Data will be analysed using thematic analysis. Thematic analysis was chosen as it allows for a theoretically flexible approach, while also being a flexible tool to analyse data in a rich and detailed way. ${ }^{44}$ Thematic analysis will be carried out in six phases: familiarising oneself with the data, generating initial codes, searching for themes, reviewing themes, defining and naming themes and finally, compiling a report of the analysis. ${ }^{44}$ Themes will 
be derived from the data. Data saturation is a complex element of qualitative research and one that has been the focus of much debate and discussion. ${ }^{45-47}$ While it is somewhat difficult to determine sample size for qualitative research a priori, it is nonetheless an increasingly important element of quality control. The initial analysis sample will have in total 12 interviews. This number was deemed adequate from previous research into the most likely point at which data saturation is reached, given a relatively homogenous sample. ${ }^{47}$ The stopping criterion will be set at further three interviews, in order to ensure that data saturation has been reached. ${ }^{45}$ Analyses will be facilitated by the use of NVIVO 11 to organise the data. AS will primarily code the interview transcripts to generate overarching themes to analyse and interpret the data. A second researcher will crosscheck codes generated by AS for a number of interviews in order to ensure credibility and consistency. A description of the coding tree will be provided for transparency. Quotations will be presented, together with the corresponding participant pseudonym to illustrate how each theme was derived from the data. Major themes and their related subordinate themes will also be illustrated. Owing to the lack of standardised guidelines to report mixed-methods protocols, the Consolidated Criteria for Reporting Qualitative Research (COREQ) checklist $^{48}$ will be used. This checklist was chosen as the qualitative component is the primary focus of this research. ${ }^{48}$ It was not deemed appropriate to return transcripts to participants for comments or corrections due to the highly sensitive nature of the research topic.

\section{Validity and reflexivity}

Discordant findings or those that run contrary to generated themes will also be presented. ${ }^{49}$ The first author of this protocol (AS) will conduct all of the interviews for this study. She is a PhD student, with a Masters in Public Health and is experienced in the area of mental health research. She has received specialised inhouse training from the NSRF in preparation for conducting these interviews. This training included how to communicate with people at risk, understanding the risk and protective factors associated with suicide and identifying and effectively dealing with someone at risk of suicide. The researcher receives weekly supervision from the PI, EA. EA is a trained psychotherapist who is specialised in suicidology and can assess individual cases if necessary. EA will undertake any clinical needs assessment with participants, if necessary, in consultation with AS.

\section{DISSEMINATION}

\section{Respect for autonomy}

Participants have given their written permission as part of the previous SSIS-ACE study to be reapproached in the future by a member of the research team. Prior to the initiation of the interview, it is explained that this study is a follow-up to the SSIS-ACE study that they previously took part in. The reasons for carrying out this research are explained to participants. It is explained to participants that confidentiality will be maintained within the research team, but will be broken in certain circumstances, including disclosure of child sexual abuse or threats to harm oneself or someone else. Before initiation of any interview, the participant is asked to carefully read through a detailed participant information sheet. They are if they understand the detailed information sheet and any questions they have are answered by the researcher. They are then be asked to complete a consent form and given a copy of this to keep. The minimum age for a participant is 18 years of age.

While those who are approached to participate in this study have given consent to future contact, it is clearly explained to them that there is no obligation to participate. In addition, it is highlighted that they are free to withdraw from the study at any time. Each person in the study will be offered facilitation of support by AS in consultation with the PI.

\section{Beneficence and non-maleficence}

Owing to family history of suicidal behaviour, participants are at increased risk of suicidal behaviour themselves. ${ }^{50}$ AS has undertaken specialised inhouse training from the NSRF based in Cork, in relation to responding to distress and risk in the context of telephone and face-to-face contact with participants and in identifying the indicators of acute suicidality. If necessary, AS can facilitate assessment by a psychotherapist (PI; EA).

For family informants bereaved by suicide, being approached to take part in a research study may be perceived as positive. ${ }^{29} 51{ }^{52}$ Benefits include facilitation of additional support when required, and the opportunity to discuss their relative with a trained interviewer in a supportive atmosphere. The family informants may also derive satisfaction from contributing to the evidence surrounding the experiences and support needs of people bereaved by suicide. A recent study of interviews with vulnerable populations explored experiences of participating in suicide and self-harm research. They found that participants were far more likely to derive benefit from participation than to experience negative effects. ${ }^{53}$ A recent systematic review sought to investigate the commonly held perception that asking about suicidality in either a research or clinical setting can increase suicidal tendencies. The review concluded that acknowledging and talking about suicide can actually reduce, rather than increase suicidal ideation. Moreover, talking about suicide and suicidal ideation may lead to an improvement in mental health in treatment-seeking populations. ${ }^{54}$

\section{DISSEMINATION}

The findings of this research will be disseminated to regional, national and international audiences through 
publication in peer-reviewed international journals and presentations at scientific conferences. The authors will produce one main mixed-methods outcome paper from this study on the analysis proposed, with the possibility of another paper exploring the impact of the inquest process on family members, depending on time constraints. This study also forms part of a $\mathrm{PhD}$ thesis for AS.

\section{CONCLUSION}

The main aim of this research is to examine how family members have been physically and psychologically affected by a relative's suicide, while also providing an indepth description of the impact of the death on their personal, professional, familial and social lives. In addition, a description of the support service needs required by family members bereaved by suicide serves as a secondary objective of the study.

Contributors AS drafted the initial protocol document. AS, CL, EA and PC contributed to the design of the study. KM-S, PC and EA contributed to planned analyses. KM-S, CL, EA and PC contributed to revising drafts. All authors contributed to the final manuscript.

Funding This work was conducted as part of the SPHeRE Programme under grant number SPHeRE/2013/1. The funding received from the Health Research Board to conduct the original SSIS-ACE case-control study, grant number HRA-2013-PHR-438, is also acknowledged.

Competing interests None declared.

Ethics approval Clinical Research Ethics Committee of University College Cork, reference number: ECM 4 (0) 19/01/2016.

Provenance and peer review Not commissioned; externally peer reviewed.

Data sharing statement The data recorded, transcribed and analysed will be very sensitive in nature. Owing to the relatively small number of participants and the specific geographic location, it would not be appropriate to consider data sharing due to the risk of people being potentially identified.

Open Access This is an Open Access article distributed in accordance with the Creative Commons Attribution Non Commercial (CC BY-NC 4.0) license, which permits others to distribute, remix, adapt, build upon this work noncommercially, and license their derivative works on different terms, provided the original work is properly cited and the use is non-commercial. See: http:// creativecommons.org/licenses/by-nc/4.0/

\section{REFERENCES}

1. Pitman A, Osborn D, King M, et al. Effects of suicide bereavement on mental health and suicide risk. Lancet Psychiatry 2014;1:86-94.

2. World Health Organisation. Preventing suicide: a global imperative. Luxemburg, 2014:89.

3. Pitman AL, Osborn DPJ, Rantell K, et al. Bereavement by suicide as a risk factor for suicide attempt: a cross-sectional national UK-wide study of 3432 young bereaved adults. BMJ Open 2016;6: e009948.

4. Ferrey AE, Hughes ND, Simkin S, et al. The impact of self-harm by young people on parents and families: a qualitative study. BMJ Open 2016;6:e009631.

5. Oldershaw A, Richards C, Simic M, et al. Parents' perspectives on adolescent self-harm: qualitative study. $\mathrm{Br} J$ Psychiatry 2008;193:140-4.

6. Bolton JM, Au W, Leslie WD, et al. Parents bereaved by offspring suicide: a population-based longitudinal case-control study. JAMA Psychiatry 2013;70:158-67.

7. Huang J, Valdimarsdóttir U, Fall K, et al. Pancreatic cancer risk after loss of a child: a register-based study in Sweden during 1991-2009. Am J Epidemiol 2013;178:582-9.
8. Sin NL. The protective role of positive well-being in cardiovascular disease: review of current evidence, mechanisms, and clinical implications. Curr Cardiol Rep 2016;18:106.

9. Whooley MA, Wong JM. Depression and cardiovascular disorders Annu Rev Clin Psychol 2013;9:327-54.

10. Tosevski DL, Milovancevic MP. Stressful life events and physical health. Curr Opin Psychiatry 2006;19:184-9.

11. Stroebe M, Schut H, Stroebe W. Health outcomes of bereavement. Lancet 2007;370:1960-73.

12. de Groot MH, Keijser Jd, Neeleman J. Grief shortly after suicide and natural death: a comparative study among spouses and first-degree relatives. Suicide Life Threat Behav 2006;36:418-31.

13. Weinberg RJ, Dietz LJ, Stoyak S, et al. A prospective study of parentally bereaved youth, caregiver depression, and body mass index. J Clin Psychiatry 2013;74:834-40.

14. Wilcox $\mathrm{H}$, Mittendorfer-Rutz $\mathrm{E}$, Kjeldgård L, et al. Functional impairment due to bereavement after the death of adolescent or young adult offspring in a national population study of $1,051,515$ parents. Soc Psychiatry Psychiatr Epidemiol 2015;50:1249-56.

15. Xu G, Li N. A comparison study on mental health status between suicide survivors and survivors of accidental deaths in rural China. J Psychiatr Ment Health Nurs 2014;21:859-65.

16. Hjelmeland $\mathrm{H}$, Knizek $\mathrm{BL}$. Why we need qualitative research in suicidology. Suicide Life Threat Behav 2010;40:74-80.

17. Brent $\mathrm{D}$, Melhem N, Donohoe MB, et al. The incidence and course of depression in bereaved youth 21 months after the loss of a parent to suicide, accident, or sudden natural death. Am J Psychiatry 2009;166:786-94.

18. Sveen CA, Walby FA. Suicide survivors' mental health and grief reactions: a systematic review of controlled studies. Suicide Life Threat Behav 2008;38:13-29.

19. Hanschmidt F, Lehnig F, Riedel-Heller SG, et al. The stigma of suicide survivorship and related consequences-a systematic review. PLoS ONE 2016;11:e0162688.

20. Pitman AL, Osborn DP, Rantell K, et al. The stigma perceived by people bereaved by suicide and other sudden deaths: a cross-sectional UK study of 3432 bereaved adults. J Psychosom Res 2016;87:22-9.

21. Sugrue JL, McGilloway S, Keegan O. The experiences of mothers bereaved by suicide: an exploratory study. Death Stud 2013;38:118-24.

22. Peters K, Cunningham C, Murphy G, et al. 'People look down on you when you tell them how he died': qualitative insights into stigma as experienced by suicide survivors. Int J Ment Health Nurs 2016;25:251-7.

23. Owens $\mathrm{C}$, Lambert $\mathrm{H}$, Lloyd $\mathrm{K}$, et al. Tales of biographical disintegration: how parents make sense of their sons' suicides. Sociol Health IIIn 2008;30:237-54.

24. Maple M, Edwards H, Plummer D, et al. Silenced voices: hearing the stories of parents bereaved through the suicide death of a young adult child. Health Soc Care Community 2010;18:241-8.

25. Trimble T, Hannigan B, Gaffney M. Suicide postvention; coping, support and transformation. Ir J Psychol 2012;33:115-21.

26. Murphy SA, Johnson LC. Finding meaning in a child's violent death: a five-year prospective analysis of parents' personal narratives and empirical data. Death Stud 2003;27:381-404.

27. Currier JM, Holland JM, Neimeyer RA. Sense-making, grief, and the experience of violent loss: toward a mediational model. Death Stud 2006;30:403-28.

28. Neimeyer RA, Prigerson HG, Davies B. Mourning and meaning. Am Behav Sci 2002;46:235-51.

29. Dyregrov KM, Dieserud G, Hjelmeland HM, et al. Meaning-making through psychological autopsy interviews: the value of participating in qualitative research for those bereaved by suicide. Death Stud 2011;35:685-710.

30. Smith A, Joseph S, Das Nair R. An interpretative phenomenological analysis of posttraumatic growth in adults bereaved by suicide. $J$ Loss Trauma 2011:16:413-30.

31. Linley PA, Joseph S. Positive change following trauma and adversity: a review. J Trauma Stress 2004;17:11-21.

32. Feigelman W, Jordan JR, Gorman BS. Personal growth after a suicide loss: cross-sectional findings suggest growth after loss may be associated with better mental health among survivors. Omega (Westport) 2009;59:181-202.

33. Lindqvist $P$, Johansson $L$, Karlsson $U$. In the aftermath of teenage suicide: a qualitative study of the psychosocial consequences for the surviving family members. BMC Psychiatry 2008;8:26.

34. Johnson RB, Onwuegbuzie AJ. Mixed methods research: a research paradigm whose time has come. Educ Res 2004;33:14-26. 
35. Lyons E, Coyle A. Analysing qualitative data in psychology. 2nd edn. London: SAGE, 2016.

36. Neimeyer RA, Klass D, Dennis MR. A social constructionist account of grief: loss and the narration of meaning. Death Stud 2014;38:485-98

37. Gunnell D, Bennewith O, Simkin S, et al. Time trends in coroners' use of different verdicts for possible suicides and their impact on officially reported incidence of suicide in England: 1990-2005. Psychol Med 2013;43:1415-22.

38. Palmer BS, Bennewith O, Simkin S, et al. Factors influencing coroners' verdicts: an analysis of verdicts given in 12 coroners' districts to researcher-defined suicides in England in 2005. J Public Health (Oxf) 2015;37:157-65.

39. Dennis $\mathrm{M}$, Read $\mathrm{S}$, Andrews $\mathrm{H}$, et al. Suicide in a single health district: epidemiology, and involvement of psychiatric services. $J$ Ment Health 2001;10:673-82.

40. Rosenberg ML, Davidson LE, Smith JC, et al. Operational criteria for the determination of suicide. J Forensic Sci 1988;33:1445-56.

41. Lovibond PF, Lovibond SH. The structure of negative emotional states: comparison of the Depression Anxiety Stress Scales (DASS) with the Beck Depression and Anxiety Inventories. Behav Res Ther 1995;33:335-43.

42. Brown TA, Chorpita BF, Korotitsch W, et al. Psychometric properties of the Depression Anxiety Stress Scales (DASS) in clinical samples. Behav Res Ther 1997;35:79-89.

43. Antony MM, Bieling PJ, Cox BJ, et al. Psychometric properties of the 42-item and 21-item versions of the Depression Anxiety Stress Scales in clinical groups and a community sample. Psychol Assess 1998:10:176-81.

44. Braun V, Clarke V. Using thematic analysis in psychology. Qual Res Psychol 2006;3:77-101.
45. Francis $\mathrm{JJ}$, Johnston $\mathrm{M}$, Robertson $\mathrm{C}$, et al. What is an adequate sample size? Operationalising data saturation for theory-based interview studies. Psychol Health 2010;25:1229-45.

46. O'Reilly M, Parker N. 'Unsatisfactory Saturation': a critical exploration of the notion of saturated sample sizes in qualitative research. Qual Res 2013;13:190-7.

47. Guest G, Bunce A, Johnson L. How many interviews are enough? an experiment with data saturation and variability. Field Methods 2006;18:59-82.

48. Tong A, Sainsbury P, Craig J. Consolidated criteria for reporting qualitative research (COREQ): a 32-item checklist for interviews and focus groups. Int J Qual Health Care 2007;19:349-57.

49. Sullivan GM, Sargeant J. Qualities of qualitative research: Part I. $J$ Grad Med Educ 2011;3:449-52.

50. Lopez-Castroman J, Guillaume S, Olie E, et al. The additive effect on suicidality of family history of suicidal behavior and early traumatic experiences. Arch Suicide Res 2015;19:275-83.

51. Hawton K, Houston K, Malmbergand A, et al. Psychological autopsy interviews in suicide research: the reactions of informants. Arch Suicide Res 2003;7:73-82.

52. Wong PW, Chan WS, Beh PS, et al. Research participation experiences of informants of suicide and control cases: taken from a case-control psychological autopsy study of people who died by suicide. Crisis 2010;31:238-46.

53. Biddle L, Cooper J, Owen-Smith A, et al. Qualitative interviewing with vulnerable populations: individuals' experiences of participating in suicide and self-harm based research. $J$ Affect Disord 2013;145:356-62.

54. Dazzi T, Gribble R, Wessely S, et al. Does asking about suicide and related behaviours induce suicidal ideation? What is the evidence? Psychol Med 2014;44:3361-3. 